Artículo

\title{
Detección de marcadores genéticos asociados a la resistencia a patógenos en frijol ayocote de Puebla, México
}

\author{
Régulo Ruíz-Salazar ${ }^{1,2}$ \\ María Luisa Patricia Vargas-Vázquez ${ }^{3}$ \\ Sanjuana Hernández-Delgado ${ }^{1}$ \\ José Socorro Muruaga-Martínez ${ }^{3}$ \\ Netzahualcóyotl Mayek-Pérez ${ }^{1,4 \S}$
}

${ }^{1}$ Centro de Biotecnología Genómica-IPN. Boulevard del Maestro Esq. Elias Piña s/n, Col. Narciso Mendoza, Reynosa, Tamaulipas, México. CP. 88710. (sanjuana70@ hotmail.com). ${ }^{2}$ Unidad Académica Multidisciplinaria Reynosa-Aztlán-UAT. Calle 16 y Lago de Chapala s/n, Reynosa, Tamaulipas. CP. 88740. (regulo.ruiz@uat.edu.mx). ${ }^{3}$ Campo Experimental Valle de México-INIFAP. Carretera Los ReyesTexcoco km 13.5. Coatlinchán, Texcoco, México. AP. 307. CP. 56250. (patricia_vargas_mx@yahoo.com; ymuruaga@hotmail.com). ${ }^{4}$ Universidad México Americana del Norte AC. Primera s/n, Col. El Círculo, Reynosa, Tamaulipas, México. CP. 88640.

§Autor para correspondencia: nmayeklp@yahoo.com.mx.

\section{Resumen}

El frijol ayocote (Phaseolus coccineus $\mathrm{L}$. ); $2 \mathrm{n}=2 \mathrm{x}=22$ ) es una leguminosa de las zonas altas de México, tiene importancia en la alimentación humana y detección de características de mejoramiento, muestra resistencia a patógenos y a ciertos factores abióticos que ocasionan pérdidas en el cultivo de frijol. Con la premisa de identificar el germoplasma de Phaseolus con resistencia a factores adversos bióticos y abióticos, el objetivo fue determinar la resistencia a dos patógenos importantes: tizón común [Xanthomonas axonopodis pv. phaseoli (Smith)] y antracnosis [Colletotrichum lindemutianum Sacc. \& Magn (anamorfo G. lindemuthiana Shear)] con base a la identificación de secuencias caracterizadas de regiones amplificadas (SCARs). El estudio incluyó 117 accesiones colectadas en diferentes sitios de Puebla (región Carso Huasteco), mismas que se analizaron con 10 marcadores SCAR (seis marcadores para tizón común: SAP6, BAC6, SU91, LG5, R7313, R4865, cuatro para antracnosis: SAS13, SBB14, SAB3, SH18) en el Centro de Biotecnología Genómica en Reynosa, Tamaulipas. Los marcadores de resistencia a antracnosis SAS13 y SBB14 están presentes con mayor frecuencia (89 y 74\%), seguidos por los marcadores de resistencia al tizón común BAC6 y SU91 (74 y 42\%). El germoplasma de Zacapoaxtla y Tlatlauquitepec tuvo mayor frecuencia de SCARs (Zacapoaxtla: 90\% y 100\% para SAS13 y SBB14; Tlatlauquitepec: 94\% y 56\%). Las accesiones con cinco SCARs podrían aprovecharse como fuente de resistencia a enfermedades en Phaseolus y genotipos precoces y tardíos a floración y madurez fisiológica, así como variables en color de testa, tamaño de vaina y semilla, pero no se detectó asociación entre la presencia de SCARs con la morfología del frijol ayocote.

Palabras clave: Carso Huasteco, C. lindemuthianum, patógenos de Phaseolus y Xanthomonas axonopodis pv. Phaseoli.

Recibido: agosto de 2019

Aceptado: octubre de 2019 


\section{Introducción}

El frijol ayocote (Phaseolus coccineus L.) en México es utilizado principalmente como alimento e incluso en regiones de España, Holanda y Reino Unido su consumo ha sustituido al frijol $(P$. vulgaris L.) (Rodiño et al., 2006). De las especies domesticadas del género Phaseolus spp., el frijol ayocote es la segunda especie en importancia económica (Al Hassan et al., 2016). El cultivo tradicional del frijol ayocote ocurre en pequeña escala (Schwember et al., 2017). En Centroamérica, este frijol se cultiva casi exclusivamente en asociación con maíz (Zea mays L.) (Vargas-Vázquez et al., 2007).

Dado que los frijoles son un elemento importante en el auto abasto de poblaciones rurales, la variación en las formas de cultivo, uso y formas de preparación para consumo es amplia. El tizón común es una enfermedad con amplia distribución en México debido entre otras causas al monocultivo y al intercambio continuo y reutilización del grano como semilla, factores que incrementan la acumulación del inóculo primario y la dispersión de la bacteria causal (AcostaGallegos et al., 2013). Por su parte, la antracnosis también muestra amplia distribución en nuestro país y puede ser una enfermedad devastadora si se siembran variedades susceptibles y, además, ocurren las condiciones climáticas favorables para su desarrollo (Rodríguez-Guerra et al., 2006).

El frijol ayocote tiene características sobresalientes que pueden aprovecharse en programas de mejoramiento genético del frijol común tales como: sistema radical vigoroso, numerosos nudos florales, pedúnculos largos, además destaca por su resistencia a las enfermedades virales como el virus del mosaico común y el virus del mosaico dorado; las enfermedades bacterianas como el tizón de halo (Pseudomonas syringae Van Mall) y tizón común (Xanthomonas axonopodis pv phaseoli Smith.) (Duncan et al., 2006; Ruiz-Salazar et al., 2016), así como las enfermedades fungosas como la antracnosis [Colletotrichum lindemuthianum (Sacc. \& Magn) (teleomorfo Glomerella lindemuthiana Shear)] (Ruiz-Salazar et al., 2016), presenta resistencia a factores abióticos como baja temperatura y salinidad (Rodiño et al., 2006; Al Hassan et al. 2016).

El uso de la resistencia genética simple y/o múltiple a las enfermedades de importancia económica en frijol se soporta con la búsqueda constante de nuevas fuentes de resistencia, su incorporación a los programas de cruzamiento, evaluación y selección de germoplasma segregante en condiciones de campo y/o controladas; y la obtención germoplasma con resistencia a dichos factores adversos, que además muestre rendimientos de grano altos y con estabilidad en el rendimiento y calidad del grano superior (Acosta-Gallegos et al., 2007; Anaya-López et al., 2015a).

El mejoramiento genético identifica y selecciona genotipos con base en fenotipos y la posterior introgresión de los caracteres deseados para el desarrollo de germoplasma superior. Así se han mejorado prácticamente todos los rasgos agronómicos importantes: rendimiento y calidad del grano; fenología; resistencia a enfermedades, plagas, sequía o salinidad. El proceso puede prolongarse por varios años dependiendo de la genética del rasgo y la respuesta a la selección. La selección asistida por marcadores moleculares (SAMM) podría reducir tiempo y costos del proceso (Bernardo, 2008; Collard y Mackill, 2008; Xu y Crouch, 2008).

La SAMM permite identificar con rapidez germoplasma que pueda contribuir al mejoramiento de especies cultivadas de Phaseolus spp. (Svetleva et al., 2003). A diferencia de países como Estados Unidos de América, donde la SAMM apoya y acelera la incorporación de genes en el germoplasma 
de interés (Miklas et al., 2006), en México, solo se consigna una variedad liberada bajo dicho esquema ('Dalia') (Acosta-Gallegos et al., 2014), con resistencia a BCMV y BMCNV (virus del mosaico común del frijol y raíz negra o necrosis). Duncan et al. (2012) indicó; sin embargo, que utilizar un solo SCAR no es suficiente para proveer suficiente resistencia a un patógeno, sugiriendo dos o más SCARs bajo un esquema de piramidación de genes de resistencia (Anaya-López et al., 2015a, b).

El objetivo de esta investigación fue determinar la resistencia o susceptibilidad a dos patógenos importantes de Phaseolus: tizón común [Xanthomonas axonopodis pv. phaseoli (Smith)] y antracnosis [Colletotrichum lindemutianum Sacc. \& Magn (anamorfo Glomerella lindemuthiana Shear)] en base a la identificación de secuencias caracterizadas de regiones amplificadas (SCARs).

\section{Materiales y métodos}

\section{Extracción de ADN}

El presente trabajo se realizó en el Laboratorio de Biotecnología Vegetal del Centro de Biotecnología Genómica del Instituto Politécnico Nacional (CBG-IPN) en Reynosa, Tamaulipas donde se analizaron 117 accesiones de $P$. coccineus L. colectadas en la Subprovincia Carso Huasteco de Puebla, más cinco accesiones empleadas como grupo testigo (Cuadro 1), la extracción del ADN se llevó a cabo de acuerdo con el protocolo de Doyle y Doyle (1987).

Cuadro 1. Procedencia y número de muestras de las poblaciones de Phaseolus spp. estudiadas.

\begin{tabular}{cccc}
\hline Especie & $\begin{array}{c}\text { Número de } \\
\text { accesiones }\end{array}$ & Localidad & Estado \\
\hline P. coccineus & 38 & Zacapoaxtla & Puebla \\
& 3 & Zacatlán & \\
& 18 & Tlatlauquitepec & \\
& 7 & Nauzontla & \\
& 4 & Tételes de Ávila Castillo & \\
& 4 & Zoquiapan & \\
& 6 & Huauchinango & \\
P. glabellus, $P$. vulgaris (Pinto Villa & 3 & Chignahuapan & \\
y Pinto Zapata) y P. coccineus $(P$. & 2 & Ahuacatlán & Xochiapulco \\
coccineus tipo y Blanco Tlaxcala) & 14 & Mercado de Zacapoaxtla & México \\
\hline
\end{tabular}




\section{Amplificación de marcadores SCAR}

A partir de las secuencias reportadas por el programa de mejoramiento de frijol (BIC, 2010) se seleccionaron diez marcadores SCAR de los cuales seis detectan secuencias de genes que confieren resistencia a tizón común (SAP6, BAC6, SU91, LG5, R7313 y R4865), y cuatro a la antracnosis (SAS13, SBB14, SAB3 y SH18) en frijol (Cuadro 2). Las amplificaciones se llevaron a cabo mediante un termociclador modelo GeneAmp PCR System 9700 (Applied Biosystems ${ }^{\circledR}$ ) de acuerdo con las indicaciones citadas para cada uno de los marcadores SCAR (BIC, 2010).

Cuadro 2. Información de los marcadores SCAR evaluados en frijol ayocote.

\begin{tabular}{|c|c|c|c|c|}
\hline SCAR & $\begin{array}{l}\text { Gen de } \\
\text { resistencia }\end{array}$ & $\mathrm{Pb}^{\Delta}$ & Secuencia & Referencia \\
\hline \multicolumn{5}{|c|}{ SCARs para antracnosis } \\
\hline SAS13 & $\mathrm{Co}-4^{2}$ & 950 & $\begin{array}{l}\text { F-CAC GGA CCG AAT AAG CCA CCA ACA } \\
\text { R-CAC GGA CCG AGG ATA CAG TGA AAG }\end{array}$ & \multirow{2}{*}{$\begin{array}{l}\text { Young et al. } \\
\text { (1998); Kelly et } \\
\text { al. (2003) } \\
\text { Awale y Kelly } \\
\text { (2001); Kelly et } \\
\text { al. (2003) }\end{array}$} \\
\hline SBB14 & $\mathrm{Co}-4^{2}$ & 1150 & $\begin{array}{l}\text { F-GTG GGA CCT GTT CAA GAA TAA TAC } \\
\text { R-GTG GGA CCT GGG TAG TGT AGA AAT }\end{array}$ & \\
\hline SAB3 & Co-5 & 400 & $\begin{array}{l}\text { F-TGG CGC ACA CAT AAG TTC TCA CGG } \\
\text { R-TGG CGC ACA CCA TCA AAA AAG GTT }\end{array}$ & $\begin{array}{l}\text { Vallejo y Kelly } \\
(2001)\end{array}$ \\
\hline SH18 & Co-4 & 1100 & $\begin{array}{l}\text { F-CCA GAA GGA GCT GAT AGT ACT CCA CAA C } \\
\text { R-GGT AGG CAC ACT GAT GAA TCT CAT GTT } \\
\text { GGG }\end{array}$ & $\begin{array}{l}\text { Awale y Kelly } \\
\text { (2001); Kelly et } \\
\text { al. (2003) }\end{array}$ \\
\hline \multicolumn{5}{|c|}{ SCARs para tizón común } \\
\hline SAP6 & $\begin{array}{l}\text { QTL en GL } 10 \\
\text { (GN\#1 sel. 27) }\end{array}$ & 820 & $\begin{array}{l}\text { F-GTC ACG TCT CCT TAA TAG TA } \\
\text { R-GTC ACG TCT CAA TAG GCA AA }\end{array}$ & $\begin{array}{l}\text { Miklas et al. } \\
(2000)\end{array}$ \\
\hline BAC6 & $\begin{array}{l}\text { QTL en GL } 10 \\
\text { (GN\#1 sel. 27) }\end{array}$ & 1250 & $\begin{array}{l}\text { F-TAG GCG GCG GCG CAC GTT TTG } \\
\text { R-TAG GCG GCG GAA GTG GCG GTG }\end{array}$ & Jung et al. (1999) \\
\hline SU91 & $\begin{array}{l}\text { QTL en GL } 8 \\
\text { (XAN 159) }\end{array}$ & 700 & $\begin{array}{l}\text { F-CCA CAT CGG TTA ACA TGA GT } \\
\text { R-CCA CAT CGG TGT CAA CGT GA }\end{array}$ & $\begin{array}{l}\text { Pedraza et al. } \\
\text { (1997) }\end{array}$ \\
\hline LG5 & $\begin{array}{l}\text { QTL en GL } 6 \\
\text { (XAN 159) }\end{array}$ & 900 & $\begin{array}{l}\text { F-GCA GGG TTC GAA GAC ACA CTG G } \\
\text { R-GCA GGG TTC GCC CAA TAA CG }\end{array}$ & Yu et al. (2000) \\
\hline R7313 & $\begin{array}{l}\text { QTL en GL } 8 \\
(\text { OAC 88-1) }\end{array}$ & 700 & $\begin{array}{l}\text { F-ATT GTT ATC GTC GAC ACG } \\
\text { R-AAT ATT TCT GAT CAC ACG AG }\end{array}$ & $\begin{array}{l}\text { Bai et al. (1997); } \\
\text { Beattie et al. } \\
\text { (1998) }\end{array}$ \\
\hline R4865 & $\begin{array}{l}\text { QTL en GL } 8 \\
\text { (OAC 88-1) }\end{array}$ & 950 & $\begin{array}{l}\text { F-TCC AAA GCC ATT CTA GTT } \\
\text { R-CAG CTA CTT TCA AAC TGG G }\end{array}$ & $\begin{array}{l}\text { Bai et al. }(1997) \text {; } \\
\text { Beattie } \text { et al. } \\
\text { (1998) }\end{array}$ \\
\hline
\end{tabular}

${ }^{\Delta} \mathrm{pb}=$ pares de bases del producto esperado; $\mathrm{GL}=$ grupo de ligamiento.

\section{Visualización y análisis estadístico}

Los productos de cada amplificación se tiñeron con $1 \mu \mathrm{L}$ de SYBR Gold ${ }^{\circledR}$ y se separaron en gel de agarosa al $1 \%$, a 80 V y $50 \mathrm{~mA}$. Después, cada gel se visualizó con luz UV y se foto-documentó (KODAK Digital Science 1D; Rochester, NY, EUA). La revisión de geles permitió construir una matriz binaria donde $1=$ presencia y $0=$ ausencia de banda (amplicón) y que incluyó todos los SCARs amplificados en cada genotipo. El análisis de datos se desarrolló con el programa Statistica 
(StatSoft ${ }^{\mathrm{TM}}$ ) versión 7 (StatSoft Inc. 2004) y básicamente consistió en el cálculo de frecuencias, porcentajes y promedios de ocurrencia de marcadores SCAR detectados por localidad y tipo de marcador generado.

\section{Resultados y discusión}

En este estudio, los SCARs con mayor frecuencia de amplificación fueron SAS13 y SBB14 con 89 y $74 \%$, respectivamente, en el total de las accesiones evaluadas. El germoplasma de Zacapoaxtla y Tlatlauquitepec, presentó las frecuencias de detección de SCARs de resistencia a antracnosis (34 de 38 accesiones en Zacapoaxtla y 17 de 18 en Tlatlauquitepec para SAS13; 30 de 38 y 10 de 18 para SBB14), lo que sugiere que estas accesiones son candidatas para usarse como progenitores en el mejoramiento genético de Phaseolus.

A la fecha no existían reportes de la identificación de germoplasma resistente a la antracnosis en $P$. coccineus L. mediante la detección de SCARs como SAS13 y SBB14, dado que éstos sólo se han utilizado en $P$. vulgaris, pero si se habían identificado SCARs de resistencia a tizón común tales como SAP6, BAC6 o SU91 (Ruiz-Salazar et al. 2016). De las 10 secuencias genómicas SCAR probadas, sólo siete amplificaron productos de PCR en el germoplasma de frijol ayocote evaluado.

Los SCARs SAB3, SH18 y R7313 no amplificaron en ninguna accesión. Las secuencias SAS13 y SBB14 fueron seguidas, en cuanto a mayor frecuencia de amplificación por BAC6 (74\%) y SU91 (42\%), SCARs relacionados con la resistencia al tizón común (Cuadro 3). Las localidades que presentan mayor número de accesiones con SCARs para tizón común corresponden a Zacapoaxtla y Tlatlauquitepec (25 de 38 accesiones y 11 de 18 accesiones para BAC6; para SU91 17 de 38 accesiones y 6 de 18 accesiones, respectivamente) (Cuadro 3). La mayor proporción de marcadores SCAR en germoplasma de Zacapoaxtla y Tlatlauquitepec coincide para el caso de tizón común, aunado a la identificación de SCARs asociados a la resistencia a virosis (virus del mosaico común y del mosaico dorado) y mancha angular (Phaeoisariopsis griseola) (Ruiz-Salazar et al. 2016).

Cuadro 3. Frecuencias de amplificación de SCAR en frijol ayocote de 16 localidades de Puebla, México.

\begin{tabular}{|c|c|c|c|c|c|c|c|c|c|}
\hline \multirow{2}{*}{ Localidad } & \multirow{2}{*}{$\mathrm{n}$} & \multicolumn{2}{|c|}{ Antracnosis } & \multicolumn{5}{|c|}{ Tizón común } & \multirow{2}{*}{$\bar{X}$} \\
\hline & & SAS13 & SBB14 & SAP6 & BAC 6 & SU91 & LG5 & R4865 & \\
\hline Zacapoaxtla & 38 & 34 & 30 & 0 & 25 & 17 & 2 & 15 & 17.5 \\
\hline Zacatlán & 3 & 2 & 3 & 0 & 0 & 0 & 3 & 1 & 1.2 \\
\hline Tlatlauquitepec & 18 & 17 & 10 & 0 & 11 & 6 & 2 & 5 & 7.2 \\
\hline Nauzontla & 7 & 6 & 5 & 0 & 5 & 2 & 3 & 4 & 3.5 \\
\hline Teteles de Ávila Castillo & 1 & 1 & 1 & 0 & 1 & 1 & 0 & 0 & 0.5 \\
\hline Zoquiapan & 4 & 4 & 4 & 0 & 3 & 3 & 0 & 0 & 2 \\
\hline Huauchinango & 1 & 1 & 1 & 0 & 1 & 1 & 1 & 1 & 0.8 \\
\hline Chignahuapan & 6 & 6 & 5 & 0 & 3 & 1 & 0 & 0 & 2.1 \\
\hline Ahuacatlán & 3 & 3 & 1 & 0 & 3 & 1 & 0 & 1 & 1.2 \\
\hline
\end{tabular}




\begin{tabular}{|c|c|c|c|c|c|c|c|c|c|}
\hline \multirow{2}{*}{ Localidad } & \multirow{2}{*}{$\mathrm{n}$} & \multicolumn{2}{|c|}{ Antracnosis } & \multicolumn{5}{|c|}{ Tizón común } & \multirow{2}{*}{$\overline{\mathrm{X}}$} \\
\hline & & SAS13 & SBB14 & SAP6 & BAC 6 & SU91 & LG5 & $\mathrm{R} 4865$ & \\
\hline Xochiapulco & 2 & 2 & 1 & 0 & 1 & 1 & 0 & 0 & 0.7 \\
\hline Mercado de Zacapoaxtla & 14 & 14 & 13 & 1 & 12 & 5 & 2 & 6 & 7.5 \\
\hline Mercado de Cuetzalán & 4 & 1 & 4 & 0 & 2 & 2 & 0 & 2 & 1.5 \\
\hline Mercado de Tlatlauquitepec & 6 & 6 & 5 & 0 & 6 & 5 & 0 & 2 & 3.4 \\
\hline Mercado Serdán & 6 & 6 & 2 & 0 & 3 & 0 & 0 & 1 & 1.7 \\
\hline Testigos & 5 & 3 & 3 & 0 & 4 & 3 & 1 & 0 & 2 \\
\hline Atempan & 4 & 3 & 3 & 0 & 2 & 4 & 0 & 1 & 1.8 \\
\hline Total & 122 & 109 & 91 & 1 & 82 & 52 & 14 & 37 & - \\
\hline
\end{tabular}

$\mathrm{n}=$ número de accesiones por localidad; $\overline{\mathrm{X}}=$ promedio de SCAR amplificados por localidad.

Se detectaron varias accesiones que cuentan con al menos cinco SCARs de resistencia, lo que pone de manifiesto la importancia de estas accesiones para mejoramiento genético en frijol común. Este germoplasma tiene mayor posibilidad de aprovecharse como fuente de resistencia múltiple a enfermedades en Phaseolus (Rodríguez-Guerra et al., 2006).

A pesar de las altas frecuencias de detección de SCARs en ayocotes de localidades específicas del Carso Huasteco de Puebla, debe considerarse que además de la ratificación de la resistencia efectiva en condiciones de campo en ocasiones dicha resistencia puede comportarse inestable, en virtud de la alta especificidad de la interacción entre los genotipos del patógeno y del hospedante (Rodríguez-Miranda y Rosas-Sotomayor, 2010).

Sin embargo, es notable identificar germoplasma que detenta alta frecuencia de SCARs asociados a la resistencia a dos enfermedades importantes en el centro de México, lo que sugiere que ocurren altas presiones de selección por las condiciones ambientales favorables para la mayor incidencia de enfermedades y de inóculo primario y mayor patogenicidad (Francisco-Francisco et al., 2013; Rodríguez-Guerra et al., 2006) han obligado a los agricultores de la región a seleccionar empíricamente por resistencia a enfermedades y por consiguiente, han conseguido el incremento de la frecuencia de genes de resistencia en las mismas.

México se considera centro de origen y diversidad del frijol y también los patógenos causantes de sus enfermedades exhiben amplia diversidad patogénica, como es el caso de la antracnosis (Rodríguez-Guerra et al., 206) o tizón común (Prudencio-Sains et al., 2008), Desafortunadamente, en el caso de tizón común sólo se asume la existencia de dicha variabilidad, pero no se tiene información actualizada sobre su cantidad y distribución en las regiones frijoleras de México (Navarrete y Acosta, 2000; Prudencio-Sains et al., 2008).

En el caso de la antracnosis se tiene una perspectiva más completa, pues se consignan más de 100 patotipos en el mundo, con al menos 54 presentes en México (Rodríguez-Guerra et al., 2006). La amplia variabilidad genética de los agentes causales de ambas enfermedades dificulta el desarrollo de variedades resistentes. También, por lo antes indicado es común observar que variedades con resistencia en una región, sean susceptibles en otra (Acosta-Gallegos et al., 2013). 
Las fuentes de resistencia genética al tizón común se han identificado en $P$. vulgaris, $P$. acutifolius y $P$. coccineus. Sin embargo, la mayoría de los genes de resistencia se heredan como loci de caracteres cuantitativos (QTL) y por consiguiente, exhiben variación en sus efectos genéticos debido a que están influenciados por el ambiente (Kelly et al., 2003; Miklas et al., 2006; Duncan et al., 2007). Para antracnosis, se identificó a un grupo de 21 genotipos con características diversas en cuanto a su base genética, color de grano, hábito de crecimiento y respuesta a diferentes patotipos de antracnosis en México (González-Chavira et al., 2004).

Entre ellos, destacan Pinto Villa y Bayo Mecentral 90, liberados comercialmente con resistencia a la antracnosis y que se han cultivado en diferentes regiones y condiciones en México (RodríguezGuerra et al., 2006). La mayoría de los estudios se han enfocado en la identificación de marcadores moleculares ligados a genes con efectos mayores (monogénicos) en antracnosis (Miklas, 2002). Por ejemplo, SCAR SAS 13 para antracnosis está ligado al gen de resistencia Co- $4^{2}$ que se ubica en el grupo de ligamiento 8, mientras que el SU91 es una región genómica QTL asociada con resistencia a tizón común y también se localiza en el grupo de ligamiento 8.

Entonces ambos marcadores podrían ser más efectivos en el mejoramiento al tener mayor probabilidad de co-segregar juntos en poblaciones de frijol segregantes. Emmalea y Kelly (2004) detectaron resistencia a 33 de 34 patotipos de $C$. lindemuthianum de nueve países de América con el uso del SCAR SAS13, en virtud de que Co- $4^{2}$ se detectó primero en la variedad diferencial Tu y también, en G-2333. Esta última, también denominada 'Colorado de Teopisca' es originaria de Chiapas, México, fue resistente a 380 aislamientos de antracnosis provenientes de once países de Latinoamérica por contar con los genes Co- $4^{2}$, Co-5 y Co-7 (Pastor-Corrales et al., 1994; Vallejo y Kelly, 2009).

Los SCARs SAP6, SU91 y LG5, a pesar de estar ubicados en grupos de ligamiento distintos, pueden aplicarse en la búsqueda de resistencia al tizón común. Ibarra-Pérez y Kelly (2005) asociaron SU91 a la resistencia en campo, demostrando las ventajas que podría proveer la SAMM para detectar resistencia a enfermedades en Phaseolus. La SAMM, en el desarrollo de germoplasma mejorado con resistencia a enfermedades, ha tenido éxito porque permite la selección indirecta de características deseables en comparación con las técnicas convencionales.

Park y Yu (2004) identificaron el SCAR SAS13 (ligado al gen Co-4²,0.39 cM) en las líneas de frijol H4514 Navy, H4628 Dark Red Kidney, H4642 Navy y H4836 Dark Red Kidney, obteniendo un incremento en la resistencia antracnosis de 43\% (H4642 Navy), en comparación con H4628 (29\%), H4514 (7\%) y H4836 (7\%), que resultaron susceptibles. Mukeshimana y Kelly (2003) estudiaron germoplasma de frijol común de Ruanda y solo detectaron el SCAR SH18 en la línea RWV167.

Por su parte, Miklas (2002) registró bajos niveles de resistencia a tizón común en frijol ayocote, pero altos niveles de resistencia en frijol tépary ( $P$. acutifolius A. Gray) con el SCAR R7313, mismo que no amplificó en el germoplasma de este trabajo. Según Márquez et al. (2007), dicho SCAR sólo se ha detectado en la línea XAN159 y en frijol Tépary. Dada la importancia económica del tizón común, deben desarrollarse en el corto plazo líneas que presenten espectros amplios de resistencia a dicho patógeno. 
Las accesiones con hasta cinco o seis secuencias SCAR incluyen accesiones precoces y tardías a floración y madurez, así como diversos colores de testa, tamaño de vaina y semilla, de manera que no se encontró relación entre mayor presencia de SCARs con alguna característica morfológica en particular del germoplasma de frijol ayocote del Carso Huasteco (PrudencioSains et al., 2008; Ruiz-Salazar et al., 2016). Bitocchi et al. (2017) sugieren que la domesticación actúa sobre el incremento de la diversidad funcional en loci particulares, los cuales probablemente controlan rasgos relacionados con la expansión y adaptación a nuevas condiciones agroecológicas (Cuadro 4).

Cuadro 4. Características de accesiones de frijol ayocote con cinco o seis secuencias genómicas que confieren resistencia a antracnosis y tizón común.

\begin{tabular}{ccccccc}
\hline Accesión & Localidad & $\begin{array}{c}\text { Días a } \\
\text { floración }\end{array}$ & $\begin{array}{c}\text { Días a } \\
\text { madurez }\end{array}$ & Color de semilla & $\begin{array}{c}\text { Peso 10 } \\
\text { semillas (g) }\end{array}$ & $\begin{array}{c}\text { Longitud } \\
\text { vaina (cm) }\end{array}$ \\
\hline 8449 & Tlatlauquitepec & 52 & 111 & Blanco & 5.45 & 9.49 \\
8210 & & 77 & 131 & Beige & 4.7 & - \\
8213 & & 54 & 126 & Violeta oscuro & 5.1 & 8.3 \\
8446 & & 39 & 101 & Blanco, lila, violeta, & 7.46 & 9.66 \\
8506 & Zacapoaxtla & 62 & 115 & negro & & \\
8762 & & 46 & 139 & Beige & 4.79 & 10.05 \\
8452 & & 50 & 120 & Beige, violeta, blanco y & 7.38 & 8.9 \\
8104 & & 46 & 126 & Amarillo mostaza & 5.5 & 10.4 \\
8193 & Ciudad Serdán & 78 & 131 & Beige & 2.8 & 8.32 \\
9237 & Atempan & 69 & 120 & Violeta oscuro & 4 & 7.81 \\
\hline
\end{tabular}

En el mediano y largo plazos se vislumbran varias opciones en el mejoramiento genético del frijol en México, asistido por Biotecnología. En principio, debe mantenerse la estrategia de manejar dos o más SCARs a la vez o, mejor aún, enfatizar estrategias de piramidación de genes de resistencia (Anaya-López et al., 2015a, b). También, no debe soslayarse que la eficiencia de la SAMM puede afectarse por el grado de dominancia del marcador, el tipo de ligamiento entre marcadores moleculares y rasgos de interés, la necesidad de desarrollar pruebas de progenie para corroborar la resistencia y los cambios en la especificidad de los marcadores (tipos comerciales de grano y/o acervos genéticos, por ejemplo) (Bello et al., 2014).

Finalmente, la biotecnología progresa día a día, se desarrollan nuevas estrategias de marcadores moleculares, que para el caso de México deben evaluarse y validarse. Se trabaja con estrategias de secuenciación de nueva generación que aprovechan diferentes tipos de polimorfismos, la combinación del análisis de segregantes y sus perfiles transcripcionales, el análisis de segregantes 'in silico' y la estructuración de plataformas de alto rendimiento para el análisis genómico integral (BeanCAP, Langebio) (Bello et al., 2014; Bolger et al., 2014; Mukeshimana et al., 2014; Viteri et al., 2014; Saburido-Álvarez y Herrera-Estrella, 2015). 


\section{Conclusiones}

El germoplasma de frijol ayocote del Carso Huasteco de Puebla analizado con marcadores del tipo SCAR presentó regiones asociadas con genes de resistencia a tizón común (X. axonopodis pv. phaseoli) y a antracnosis (C. lindemuthianum), donde las accesiones de Zacapoaxtla y Tlatlauquitepec son las que cuentan con mayor frecuencia de estos marcadores.

\section{Agradecimientos}

R. Ruíz-Salazar (CVU: 229696) agradece al Consejo Nacional de Ciencia y Tecnología, al Gobierno de Tamaulipas, al programa Institucional de Formación de Investigadores del IPN y al Programa ECOES-Santander el financiamiento de sus estudios doctorales en el CBG-IPN. De igual manera agradece al Dr. Guillermo Castañón-Nájera de la Universidad Juárez Autónoma de Tabasco por el apoyo brindado. Este trabajo fue financiado por el FOMIX-Gobierno del Estado de Veracruz (proyecto 94070), CONACYT-Ciencia Básica (181756) e IPN (proyecto 1636).

\section{Literatura citada}

Acosta-Gallegos, J. A.; Kelly, J. D., and Gepts, P. 2007. Prebreeding in common bean and use of genetic diversity from wild germplasm. Crop Sci. 47(suppl. 3)44-59.

Acosta-Gallegos, J. A.; Ibarra-Pérez, F. J.; Navarrete-Maya, R.; Sánchez-García, B. M.; JiménezHernández, Y. y Mendoza-Hernández, F. M. 2013. Relación entre la reacción al tizón común y el rendimiento y peso de la semilla de frijol en riego y temporal. Rev. Mex. Ciencias Agríc. 4(5):673-685.

Acosta-Gallegos, J. A.; Montero-Tavera, V.; Jiménez-Hernández, Y.; Anaya-López, J. L. and González-Chavira, M. M. 2014. 'Dalia', a new variety of bean grain, Flor de Junio type for the central region of Mexico. Rev. Mex. Cienc. Agríc. 5(2):331-336.

Al Hassan, M.; Morosan, M.; López-Gresa, M. P.; Proens, J.; Vicente, O. and Boscaiu, M. 2016. Salinity-induced variation in biochemical markers provide insight into the mechanisms of salt tolerance in common (Phaseolus vulgaris) and runner (P. coccineus) beans. Int. J. Mol. Sci. 17(1582):1-16.

Anaya-López, J. L.; Silva-Rosales, L.; Montero-Tavera, V.; Espejel, F. y Acosta-Gallegos, J. A. 2015a. Retos y oportunidades en la selección asistida de frijol resistente a BCMV y BCMNV en México. I. Dimensión del problema. Rev. Mex. Cienc. Agríc. 6(3):453-465.

Anaya-López, J. L.; Espejel, F.; Montero-Tavera, V.; Acosta-Gallegos, J. A. y Silva-Rosales, L. 2015b. Retos y oportunidades en la selección asistida de frijol resistente a BCMV y BCMNV en México. II. Oportunidades para la selección asistida. Rev. Mex. Cienc. Agríc. 6(3):495-507.

Awale, H. E. and Kelly, J. D. 2001. Development of SCAR markers linked to Co-4 $4^{2}$ gene in common bean. Annu Rept. Bean Improv. Coop. 44:119-120.

Bai, Y.; Michaels, T. E. and Pauls, K. P. 1997. Identification of RAPD markers linked to common bacterial blight resistance genes in Phaseolus vulgaris L. Genome. 40(4):544-55.

Beattie, A.; Michaels, T. E. and Paul, K. P. 1998. An efficient reliable method to screen for common bacterial blight (CBB) resistance in Phaseolus vulgaris L. Bean Improv. Coop. 41:53-54. 
Bello, M. H.; Moghaddam, S. M.; Massoudi, M.; McClean, P. E.; Cregan, P. B. and Miklas, P. N. 2014. Application of in silico bulked segregant analysis for rapid development of markers linked to Bean common mosaic virus resistance in common beans. BMC Genomics. 15(903):1-13.

Bernardo, R. 2008. Molecular markers and selection for complex traits in plants: learning from the last 20 years. Crop Sci. 48(5):1649-1664.

BIC. 2010. Bean Improvement Cooperative. SCAR markers linked with disease resistance traits in common bean (Phaseolus vulgaris). http://arsftfbean.uprm.edu/bic/wp-content/ uploads/2018/04/SCAR_Markers_2010.pdf

Bitocchi, E.; Rau, D.; Benazzo, A.; Bellucci, E.; Goretti, D.; Biagetti, E.; Panziera, A.; Laidò, G.; Rodriguez, M.; Gioia, T.; Attene, G.; McClean, P.; Lee, R. K.; Jackson, S. A.; Bertorelle, G. and Papa, R. 2017. High level of nonsynonymous changes in common bean suggests that selection under domestication increased functional diversity at target traits. Front. in Plant Sci. 7:1-15.

Bolger, M. E.; Weisshaar, B.; Scholz, U.; Stein, N.; Usadel, B. and Mayer, F. X. 2014. Plant genome sequencing-applications for crop improvement. Curr. Op. Biotechnol. 26(1):31-37.

Collard, B. C. Y., and Mackill, D. J. 2008. Marker-assisted selection: an approach for precision plant breeding in the twenty-first century. Phil. Trans. Royal Soc. Biol. Sci. 363(1491):557-572.

Doyle, J. J. and Doyle, J. L. 1987. A rapid DNA isolation procedure for small quantities of fresh leaf tissue. Phytochem. Bull. 19(1):11-15.

Duncan, R. W.; Terán, H.; Singh, S. P. and Gilbertson, R. L. 2006. Comparison of marker-assisted and direct selection for introgression of common bacterial blight resistance in common bean. Bean Improv. Coop. 49:11-12.

Duncan, R.; Singh, S. P. and Gilbertson, R. L. 2007. Marker-assisted versus direct selection for common bacterial blight resistance in common bean. International Annual Meetings ASACSSA-SSSA. November 4-8, New Orleans, LA, USA. Abstr. 334-2. https://a-cs.confex.com/a-c-s/2007am/techprogram/index.html.

Duncan, R. W.; Gilbertson, R. L. and Singh, S. P. 2012. Direct and marker-assisted selection for resistance to common bacterial blight in common bean. Crop Sci. 52(4):1511-1521.

Emmalea, G. E. and Kelly, J. D. 2004. The Mesoamerican anthracnose resistance gene, Co-42, does not confer resistance in certain Andean genetic backgrounds. Bean Improv. Coop. 47:245-246.

Francisco-Francisco, N.; Gallegos-Morales, G.; Ochoa-Fuentes, Y. M.; Hernández-Castillo, F. D.; Benavides-Mendoza, A. y Castillo-Reyes, F. 2013. Aspectos fundamentales del tizón común bacteriano (Xanthomonas axonopodis pv. phaseoli Smith): características, patogenicidad y control. Rev. Mex. Fitopatol. 31(2):147-160.

González-Chavira, M.; Rodríguez-Guerra, R.; Hernández-Godínez, F.; Acosta-Gallegos, J. A.; Martínez de la Vega, O. and Simpson, J. 2004. Analysis of pathotypes of Colletotrichum lindemuthianum found in the central region of Mexico and resistance in elite germ plasm of Phaseolus vulgaris. Plant Dis. 88(2):152-156.

Ibarra-Pérez, F. J. and Kelly, J. D. 2005. Molecular markers used to validate reaction of elite bean breeding lines to common bacterial blight. Bean Improv. Coop. 48:98-99.

Jung, G.; Skroch, P. W.; Nienhuis, J.; Coyne, D. P.; Arnaud-Santana, E.; Ariyarathne, H. M. and Marita, J. M. 1999. Confirmation of QTL associated with common bacterial blight resistance in four different genetic backgrounds in common bean. Crop Sci. 39(5):14481455. https://doi.org/10.2135/cropsci1999.3951448x. 
Kelly, J. D.; Gepts, P.; Miklas, P. N. and Coyne, D. P. 2003. Tagging and mapping of genes and QTL and molecular marker-assisted selection for traits of economic importance in bean and cowpea. Field Crops Research. 82(2-3):135-154. https://doi.org/10.1016/S03784290(03)00034-0.

Márquez, M. L.; Terán, H. and Singh, S. P. 2007. Selecting common bean with genes of different evolutionary origins for resistance to Xanthomonas campestris pv. phaseoli. Crop Sci. 47(4):1367-1374. https://doi.org/10.2135/cropsci2006.12.0769.

Miklas, P. N.; Smith, J. R.; Riley, R.; Grafton, K. F.; Singh, S. P.; Jung, G. and Coyne, D. P. 2000. Marker-assisted breeding for pyramided resistance to common bacterial blight in common bean. Annu. Rep. Bean Improv. Coop. 43:39-40.

Miklas, P. N. 2002. Marker-assisted selection for disease resistance in common bean. Bean Improv. Coop. 45:21-22.

Miklas, P. N.; Kelly, J. D.; Beebe, S. E. and Blair, M. W. 2006. Common bean breeding for resistance against biotic and abiotic stresses: from classical to MAS breeding. Euphytica. 147(1-2):105-131.

Mukeshimana, G. and Kelly, J. D. 2003. Evaluation of Rwandan varieties for disease resistance. Bean Improv. Coop. 46:145-146.

Mukeshimana, G.; Butare, I.; Cregan, P. B.; Blair, M. W. and Kelly, J. D. 2014. Quantitative trait loci associated with drought tolerance in common bean. Crop Sci. 54(3):923-938.

Navarrete, R. y Acosta, J. 2000. Genotipos de frijol (Phaseolus vulgaris L.) resistentes a Xanthomonas campestris pv. phaseoli de México. Agron. Mesoam. 11(1):17-23.

Park, S. J. and Yu K. 2004. Molecular marker assisted selection technique in improvement of multiple disease resistance in common bean: a plant breeder's perspective. Bean Improv. Coop. 47:73-74.

Pastor-Corrales, M. A.; Erazo, O. A.; Estrada, E. I. and Singh, S. P. 1994. Inheritance of anthracnose resistance in common bean accession G 2333. Plant Dis. 78(10):959-962.

Pedraza, F.; Gallego, G.; Beebe, S. and Tohme, J. 1997. Marcadores SCAR y RAPD para la resistencia a la bacteriosis común (CBB). pp. 130-134. In: Singh, S. P. y Voysest, O. (Eds.). Taller de mejoramiento de frijol para el Siglo XXI: bases para una estrategia para América Latina. CIAT. Cali, Colombia. 559 p.

Prudencio-Sains, J. M.; Navarrete-Maya, R.; Navarrete-Maya, J. y Acosta-Gallegos, J. A. 2008. Dinámica de los tizones común y de halo del frijol en el Valle de México. Agric. Téc. Méx. 34(2):213-223.

Rodiño, A. P.; Lema, M.; Pérez, M.; Santalla, M. and De Ron, M. 2006. Assessment of runner bean (Phaseolus coccineus L.) germplasm for tolerance to low temperature during early seedling growth. Euphytica. 155(1):63-70.

Rodríguez-Guerra, R.; Acosta-Gallegos, J. A.; González-Chavira, M. M. y Simpson, J. 2006. Patotipos de Colletotrichum lindemuthianum y su implicación en la generación de cultivares resistentes de frijol. Agric. Téc. Méx. 32(1):101-114.

Rodríguez-Miranda, O. y Rosas-Sotomayor, J. C. 2010. Selección de genotipos de frijol común por su resistencia al tizón bacteriano. Agron. Mesoam. 21(2):275-280.

Ruíz-Salazar, R.; Muruaga-Martínez, J. S.; Vargas-Vázquez, M. L. P.; Alejandre-Iturbide, G.; Castañón-Nájera, G.; Hernández-Delgado, S.; Almaraz-Abarca, N. y Mayek-Pérez, N. 2016. Marcadores moleculares SCAR para identificar fuentes de resistencia a enfermedades en frijol ayocote (Phaseolus coccineus). ФYTON. Inter. J. Exp. Bot. 85:184-193. 
Saburido-Álvarez, M. S. y Herrera-Estrella, A. 2015. El frijol en la era genómica. Rev. Dig. Univ. 16(2):1-16.

Schwember, A. R.; Carrasco, B. and Gepts, P. 2017. Unraveling agronomic and genetic aspects of runner bean (Phaseolus coccineus L.). Fields Crop Res. 206(1):86-94. https://doi.org/10.1016/j.fcr.2017.02.020.

StatSoft, Inc. 2004. Statistica for Windows (computer program manual). Version 7.0 Tulsa, USA. $304 \mathrm{p}$.

Svetleva, D.; Velcheva, M. and Bhowmik, G. 2003. Biotechnology as a useful tool in common bean (Phaseolus vulgaris L.) improvement. Euphytica. 131(2):189-200. https://doi.org/10.1023/A:1023983831582.

Vallejo, V. and Kelly, J. D. 2001. Development of a SCAR marker linked to Co-5 gene in common bean. Annu Rep. Bean Improv. Coop. 44:121-122.

Vallejo, V. and Kelly, J. D. 2009. New insights into the anthracnose resistance of common bean landrace G 2333. Open Hortic. J. 2:29-33.

Vargas-Vázquez, M. L. P.; Muruaga-Martínez, J. S.; Hernández-Casillas, J. M. y Díaz-De la Cruz, J. 2007. Diagnóstico de la forma cultivada del frijol ayocote Phaseolus coccineus L. e información geográfica de los sitios de colecta. Campo Experimental Valle de México, INIFAP. Chapingo, México. 2-18 pp.

Viteri, D.; Cregan, P.; Trapp, J.; Miklas, P. N. and Singh, S. P. 2014. A new common bacterial blight resistance QTL in VAX 1 common bean and interaction of the new QTL, SAP6, and SU91 with bacterial strains. Crop Sci. 54(4):1598-1608. http://dx.doi.org/10.2135/ cropsci2014.01.0008.

$\mathrm{Xu}$, Y. and Crouch J. H. 2008. Marker-assisted selection in plant breeding: from publications to practice. Crop Sci. 48(2):391-407.

Young, R. A.; Melotto, M.; Nodari, R. O. and Kelly, J. D. 1998. Marker assisted dissection of the oligogenic anthracnose resistance in common bean cultivar, G 2333. Theor. Appl. Genet. 96(1): 87-94. https://doi.org/10.1007/s001220050713.

Yu, K.; Park, S. J. and Poysa, V. 2000. Marker-assisted selection of common beans for resistance to common bacterial blight: efficiency and economics. Plant Breed. 119(5):411-416. https://doi.org/10.1046/j.1439-0523.2000.00514.x. 